\title{
How TO UNIFY
}

\author{
NICHOLAS K. JONES \\ University of Birmingham
}

\begin{abstract}
This paper evaluates the argument for the contradictoriness of unity, that begins Priest's recent book One. The argument is seen to fail because it does not adequately differentiate between different forms of unity. This diagnosis of the argument's failure is used as a basis for two consistent accounts of unity. The paper concludes by arguing that reality contains two absolutely fundamental and unanalysable forms of unity, which are in principle presupposed by any theory of anything. These fundamental forms of unity are closely related to the unity of propositions and facts.
\end{abstract}

$\mathrm{T}$ HE emergence of unity from plurality is central to our conceptual scheme. Instances range from the familiar and mundane-for example, you are a human being composed of many organs, the European Union is an institution composed of many nation states, and the Broken Earth cycle is a work of fiction composed of three novels-to the technical and recherché-for example, the set of integers is a single individual composed of many numbers, and the proposition that Tibbles purrs is a single individual composed of Tibbles and the property of purring (or perhaps from representations thereof). The notion of composition may not be exactly the same in each case; for unlike the set of integers, you are not plausibly regarded as a value of the set-builder operation. ${ }^{1}$ Yet a single phenomenon seems nonetheless present throughout: the emergence of one whole from many parts, of unity from plurality.

Familiar as this phenomenon may be, Graham Priest's provocative recent book One begins by arguing that it is inherently contradictory. ${ }^{2}$ The core of the argument runs as follows (8-9). If some things are unified, then that is because something unifies them. Priest calls this unifying something their gluon. But

Contact: Nicholas K. Jones <n.k.jones@bham.ac.uk>

1. Fine (2010) and Hawley (2006) discuss the view that there are many different forms of composition.

2. The argument comprises Chapter 1 of Priest (2014). All unadorned page references are to this book. 
is this gluon a thing or not? On the one hand, it cannot be; for if the gluon is a thing, then it cannot unify the original things-they and the gluon together comprise just another plurality, not an individual. On the other hand it must be; for (i) I've just referred to and thought about it, and (ii) if the gluon is not a thing, then the original things are not unified because nothing unifies them. The emergence of one from many thus gives rise to a dilemma, both horns of which lead to contradiction.

I have two primary goals in this paper. One is to evaluate this argument for the inconsistency of a central component of our conceptual scheme. The other is to provide a positive, consistent account of how unity emerges from plurality. In fact, I will provide two such accounts. Along the way, I hope to provide a better understanding of what it is for relations to relate, the role of relations in accounting for composite wholes, the variety of different kinds of unity, and of what I will argue are reality's two most fundamental forms of unity, namely the unity of propositions and facts.

In more detail, I will argue that both horns of the dilemma fail. The argument of the first horn is a version of Bradley's regress, which I present in Section $1{ }^{3}$ I respond to this first horn in Section 2 by distinguishing two different notions of unity. One is the compositional unity with which we began: the emergence of one whole from many parts. The other is relationality: interrelatedness amongst things. Priest's Bradleyan regress argument shows that compositional unity cannot explain compositional unity. Yet this is consistent with the plausible and natural idea that relationality explains compositional unity, and is not itself a form of compositional unity. This observation provides the basis for my first account of unity. I respond to the second horn of the dilemma in Section 3 by presenting my second account of unity, which generalises the first so as to avoid invoking any such things as relations or gluons. This shows how, whether gluons are things or not, they can consistently explain how one emerges from many. Section 4 then discusses a revenge problem for my second proposal. I respond by differentiating compositional unity from a form of unity closely related to the unity of propositions, which I argue is one of reality's most fundamental forms of unity and cannot in principle be explained in other terms. The paper closes by identifying a second fundamental form of unity with this feature, which is closely related to the unity of facts.

\section{The Argument}

Here's how Priest argues that gluons cannot unify, if they are things: ${ }^{4}$

3. See Bradley (1897 Chapters 2 and 3) for Bradley's original version of the regress, and Perovic (2017) for a helpful overview.

4. Priest uses 'object' where I use 'thing'. 
Suppose that we have a unity comprising the parts $a, b, c, d$, for example. There must be something which, metaphysically speaking, binds them together. This is the object's gluon, $g$. But then there must be something which binds $g$ and $a, b, c, d$ together, a hyper-gluon, $g^{\prime}$. There must, then, be something which binds $g^{\prime}, g$, and $a, b, c, d$ together, a hyper-hyper-gluon, $g^{\prime \prime}$. Obviously we are off on an infinite regress. Moreover, it is a vicious one.

Perhaps it is not immediately obvious that this is so. Could there not just be a whole lot'a gluin' goin' on? To understand why this is not a valid response, we must come back to what is at issue here. Our original problem was how a unity of parts is possible. We need an explanation. Given a bunch of parts, simply invoking another object does not do this. We still have the original problem of how a unity of parts is possible.... In vicious regresses of this kind ... the infinity has, in fact, precious little to do with matters. The point is that something has already gone wrong at the first step: a failure of explanation. (II)

Although the assumption that gluons are things plays no explicit role in this passage, Priest's idea is that merely postulating and quantifying over gluons involves treating them as things: "we can refer to it [i.e., a postulated gluon], quantify over it, talk about it. If this does not make something an object [i.e., a thing], I am at a loss to know what could" (15, original emphasis). I return to this connection between quantification and thinghood in Section 3 . Until then, it is fine for present purposes to treat Priest as arguing here that an adequate account of unity is impossible because an adequate account of how gluons unify is impossible.

It will be useful to regiment Priest's argument slightly. As a first attempt, we might see it as employing the following principles: ${ }^{5}$

Unity $1 a$ For any $x_{1}, \ldots, x_{n}\left(x_{1}, \ldots, x_{n}\right.$ are unified $\rightarrow$ some $y$ unifies $\left.x_{1}, \ldots, x_{n}\right)$

Unity $2 a$ For any $x_{1}, \ldots, x_{n}, y\left(y\right.$ unifies $x_{1}, \ldots, x_{n} \rightarrow y, x_{1}, \ldots, x_{n}$ are unified $)$

We can then represent Priest as arguing as follows. Consider any $a, b, c, d$ that compose something, that is, that are unified in the sense that concerns Priest. So (a1) is true:

(a1) $a, b, c, d$ are unified.

(a2) So by Unity 1a: (for some $g$ ) $g$ unifies $a, b, c, d$.

(a3) So by Unity 2a: $g, a, b, c, d$ are unified.

5. ' $\rightarrow$ ' is the material conditional. ' $n$ ' can be any natural number. 
(a4) So by Unity 1a: (for some $g^{\prime}$ ) $g^{\prime}$ unifies $g, a, b, c, d$.

(a5) So by Unity 2a: $g^{\prime}, g, a, b, c, d$ are unified.

On this reconstruction, the argument is valid and the regress is genuine but not vicious. $^{6}$

The argument shows that if Unity $1 \mathrm{a}$ and $2 \mathrm{a}$ are true, then whenever some things compose a unity there is an infinite series of facts of the forms (i) $x_{1}, \ldots, x_{n}$ are unified, and (ii) $y$ unifies $x_{1}, \ldots, x_{n}$. That infinite series is no more problematic than this other infinite series of facts that follow from it being the case that $p: p$, it's true that $p$, it's true that it's true that $p$, it's true that it's true that it's true that $p, \ldots$ Setting qualms about infinity aside, there is no obvious reason to regard the mere existence of either series as problematic.

Unlike the quoted passage, this reconstruction makes no mention of explanation. Yet Priest's goal is an account of "how, if an object has parts, these cooperate to produce a unity-one thing" (5) and "how a unity of parts is possible" (11). This requires an explanation of how unity emerges from plurality that does not itself involve unity. ${ }^{7}$ We obtain a better reconstruction of Priest's argument by making the explanatory connections explicit. To do so, I use 'because' as a two-place sentential connective that expresses the relevant notion of explanation.

Our second reconstruction presents the argument as employing these two principles: 8

6. This reconstruction does not capture Priest's intention. I discuss it here in order to illustrate the importance of explanatory concerns in obtaining a good argument.

7. Is this explanation in some familiar sense, or the more rarefied form of metaphysical explanation popular in the recent literature about ground? I don't know. But I don't think that it's relevant for present purposes. What matters is that the explanation's goal be to render unity intelligible in unity-free terms.

8. I differentiate the principles and so present each stage of the regress as decomposing into two sub-stages, one for an application of each principle. Note, however, that Priest does not explicitly distinguish these steps in the quoted passage. An alternative reconstruction that perhaps stays closer to Priest's presentation would employ the following in place of Unity $2 \mathrm{~b}$ :

For any $x_{1}, \ldots, x_{n}, y\left(y\right.$ unifies $x_{1}, \ldots, x_{n} \rightarrow$ for some $z\left(y\right.$ unifies $x_{1}, \ldots, x_{n}$ because $z$ unifies $\left.\left.y, x_{1}, \ldots, x_{n}\right)\right)$.

The argument would then proceed beyond (b2) solely by applications of this new principle, without invoking Unity $\mathrm{Ib}$ again: $g$ unifies $a, b, c, d$ because $g^{\prime}$ unifies $g, a, b, c, d$, because $g^{\prime \prime}$ unifies $g^{\prime}, g, a, b, c, d, \ldots$. Notice that the new principle follows from Unity $1 \mathrm{~b}$ and $2 \mathrm{~b}$ by transitivity and factivity of 'because'. Thus nothing of substance that follows seems to turn on the difference between the version of the argument in the text and this alternative one. It is clear, however, that this alternative regress concerns not the notion of unity itself or of things being unified, but of one thing unifying some others. Since these are different notions-one is monadic, the other relational, for a start-this alternative argument reveals nothing distinctive 
Unity $1 b$ For any $x_{1}, \ldots, x_{n}\left(x_{1}, \ldots, x_{n}\right.$ are unified $\rightarrow$ for some $y\left(x_{1}, \ldots, x_{n}\right.$ are unified because $y$ unifies $\left.x_{1}, \ldots, x_{n}\right)$ )

Unity $2 b$ For any $x_{1}, \ldots, x_{n}, y\left(y\right.$ unifies $x_{1}, \ldots, x_{n} \rightarrow y$ unifies $x_{1}, \ldots, x_{n}$ because $y, x_{1}, \ldots, x_{n}$ are unified)

Unity $1 b$ says that when things are unified, that's because a particular thing unifies them. And Unity $2 b$ says that when one thing unifies others, that's because it and they are unified. We can now represent Priest as arguing thus, concerning any $a, b, c, d$ that compose something: ${ }^{9}$

(b1) $a, b, c, d$ are unified.

(b2) So by Unity 1 b: (for some $g$ ) $a, b, c, d$ are unified because $g$ unifies $a, b, c, d$.

(b3) So by Unity 2b: $g$ unifies $a, b, c, d$ because $g, a, b, c, d$ are unified.

(b4) So by Unity 1 b: (for some $g^{\prime}$ ) $g, a, b, c, d$ are unified because $g^{\prime}$ unifies $g, a, b, c, d$.

(b5) So by Unity $2 b: g^{\prime}$ unifies $g, a, b, c, d$ because $g^{\prime}, g, a, b, c, d$ are unified.

On this reconstruction, the argument is valid and delivers an explanatory regress:

$a, b, c, d$ are unified

$$
\downarrow \text { because }
$$

$g$ unifies $a, b, c, d \stackrel{\text { because }}{\longrightarrow} g, a, b, c, d$ are unified

$$
\begin{gathered}
\downarrow \text { because } \\
g^{\prime} \text { unifies } g, a, b, c, d \stackrel{\text { because }}{\longrightarrow} g^{\prime}, g, a, b, c, d \text { are unified } \\
\vdots \\
\downarrow \\
\downarrow
\end{gathered}
$$

This regress is vicious, given Priest's goal: an account of how unity is possible. We began with an instance of unity at (b1). That was explained by another instance

about unification itself: this regress concerns unifiers, not unification in the sense of composing a whole. Since the argument in the text invokes unification-i.e., composition of a whole-after 'because' at every other stage, it is a better candidate for revealing something distinctive about that, as Priest seems to take it to do.

9. The validity of the argument beyond b2 requires the factivity of 'because': $\square$ (( $p$ because $q) \rightarrow(p \wedge q))$. 
of unity at (b3) (via b2). And that was explained by another instance of unity at (b5) (via b4). And so on ad infinitum. Because each instance of unity is explained by another instance of unity, unity itself goes unexplained. As an account of unity itself-the general phenomenon as opposed to particular instances-this approach therefore fails. ${ }^{10}$

Priest concludes that an adequate account of unity is impossible (if gluons/unifiers are objects). That conclusion follows only if no alternative account is available. The next section argues that by distinguishing between different kinds of unity, we can attain an adequate account of Priest's target kind of unity.

\section{Two Kinds of Unity}

Our first kind of unity is compositionality. This kind of unity is involved when a plurality compose a single individual, as in the examples with which we began. It is also Priest's target notion of unity, as his discussion throughout Chapter 1 makes clear. Two pieces of terminology will be helpful below. The first is monadic: ${ }^{11} x_{1}, \ldots, x_{n}$ are compositionally unified iff they compose something. The second is polyadic: $y$ compositionally unifies $x_{1}, \ldots, x_{n}$ iff $x_{1}, \ldots, x_{n}$ compose $y_{.}{ }^{12}$

Our second kind of unity is relationality. This kind of unity is involved when a plurality are related to one another. Like compositional unity, examples range from the mundane to the highly theoretical; for example, Tibbles is on the mat, $i$ is greater than $-i$, and $a$ grounds $b$. Again, two pieces of terminology will be helpful. The first is monadic: $x_{1}, \ldots, x_{n}$ are relationally unified iff they are related to one another. The second is polyadic: relation y relationally unifies $x_{1}, \ldots, x_{n}$

10. One might be tempted to conclude that the attempted explanation of unification failed because it started in the wrong place; to explain unification in general, we need to start by explaining not some particular instance of unification, as in (b1), but why there are any unified things at all. Tempting as this idea may be, I think it cannot be quite right. One way to explain why there are any $F$ s at all is to pick some particular instances of $F$ and then explain in completely $F$-free terms, and without presupposing any $F$-facts whatsoever, why they are $F$. As I will argue in Section 2 and Section 3. this can be done for unification, by rejecting certain versions of Unity $1 b$ and $2 b$ and replacing them with others. The problem with Unity $1 b$ and $2 b$ is not that they explain only particular instances of unification. The problem is that they jointly preclude explanation of unification in unification-free terms without presupposing any unification-facts: since Unity $\mathrm{ib}$ uses unifiers to explain unification, and Unity $2 b$ uses unification to explain unifiers, Unity $1 b^{\prime}$ 's explanation of unification is not ultimately unification-free.

11. Monadic in the sense of having one (plural) argument position, not in the sense of applying only to single individuals.

12. The precise adicity of 'compositionally unifies' depends on how we treat ' $x_{1}, \ldots, x_{n}$ '. If it is a single (ordered) plural term, then 'compositionally unifies' is dyadic. If it is a sequence of singular terms, 'compositionally unifies' is variably polyadic. Nothing turns on this issue here. Likewise mutatis mutandis for 'relationally unifies' below. 
iff $y$ is a relation that holds amongst $x_{1}, \ldots, x_{n}$ (in that order). ${ }^{13}$ So, for example, Tibbles and the mat are relationally unified because Tibbles is on the mat, and the relation of being on relationally unifies Tibbles and the mat because it holds from the former to the latter. ${ }^{14}$

These two kinds of unity are distinct: relationality is not a form of compositionality. This makes relationality available for non-regressive explanation of compositionality in other terms, as we will see below. To see why relationality is not a form of compositionality, note that when Tibbles is on the mat, it doesn't follow that there's a further thing-for example, a fact or state of affairs in the mould of Armstrong (1996) — composed of Tibbles and the mat (or even of Tibbles, the mat, and being on). One could of course postulate such a thing within one's substantive metaphysical theory of relationality. But its existence is not built into the notion of relationality itself: relationality does not, in and of itself, involve the emergence of one from many. As we will see towards the end of this section, Priest himself acknowledges this fact.

With this distinction to hand, let us return to Priest's argument. The argument is supposed to show that no adequate explanation of unity is possible. We now need to be clear about which forms of unity are at work in its three premises: (b1), Unity 1 b, and Unity $2 b$. Different versions of those premises are obtained by replacing the generic notion of unity with our two specific kinds of unity, that is, compositionality and relationality. We can block the argument by showing that some plausible distribution of truth-values across those principles (a) does not give rise to problematic regress, and (b) suffices to explain Priest's target notion of unity. And that is what I will do. To aid readability, I often use 'c-unified' and 'r-unified' (and their cognates) to abbreviate 'compositionally unified' and 'relationally unified' (and their cognates).

Let us begin with the first premise $\left(\mathrm{a}_{1}\right) /\left(\mathrm{b}_{1}\right)$. The goal is to explain how one emerges from many, that is, compositionality. So the first premise must concern c-unification:

(1) $a, b, c, d$ are c-unified.

13. Note that nominalism about relations is compatible with relational unification but not with relational unifiers. That's because relational unifiers are relations, which nominalists reject. But nominalists don't deny that things can be related to one another, as when Tibbles is on the mat, for example. Nominalists only deny that when things are related, a relation holds between them. I return to nominalism and realism about relations in Section 3.

14. Following Lewis (1983), we can distinguish between sparse and abundant notions of relational unification and unifiers. Sparse conceptions restrict the definitions in the text to highly natural or fundamental relations. For example, electron pairs with opposite spin are arguably sparsely relationally unified, and the highly natural relation of opposite spin sparsely unifies them. On abundant conceptions, by contrast, any way of being related suffices, however unnatural and gerrymandered. I'll work with sparse notions for simplicity, and aim to explicitly highlight where the distinction matters. 
This follows from the supposition that $a, b, c, d$ compose something.

(1) concerns c-unification. So a valid argument and explanation of c-unification requires a version of Unity 1 whose antecedent also concerns c-unification. But what about its consequent? There are two versions, one for c-unification and one for r-unification: ${ }^{15}$

Unity 1 ICC For any $x_{1}, \ldots, x_{n}\left(x_{1}, \ldots, x_{n}\right.$ are c-unified $\rightarrow$ for some $y\left(x_{1}, \ldots, x_{n}\right.$ are c-unified because $y$ c-unifies $\left.x_{1}, \ldots, x_{n}\right)$ )

Unity 1 cr For any $x_{1}, \ldots, x_{n}\left(x_{1}, \ldots, x_{n}\right.$ are c-unified $\rightarrow$ for some $y\left(x_{1}, \ldots, x_{n}\right.$ are c-unified because $y$ r-unifies $\left.x_{1}, \ldots, x_{n}\right)$ )

Both principles explain a certain monadic feature of pluralities-a property possessed by all and only pluralities that compose something-in terms of how they're related to a particular entity. According to Unity 1Cc, they compose it. According to Unity $1 \mathrm{cr}$, it is a relation that holds amongst them. I examine these ideas in turn.

Begin with Unity 1cc. It validates the argument from (1) to (2cc): ${ }^{16}$

(2cc) $a, b, c, d$ are c-unified because $g$ c-unifies $a, b, c, d$.

This uses c-unifiers to explain c-unification. This explanation remains within the compositional. So a further principle is required to explain c-unifiers. We need a version of Unity 2 whose antecedent concerns c-unifiers, of which there are two: ${ }^{17}$

Unity $2 c c$ For any $x_{1}, \ldots, x_{n}, y$ (y c-unifies $x_{1}, \ldots, x_{n} \rightarrow y$ c-unifies $x_{1}, \ldots, x_{n}$ because $y, x_{1}, \ldots, x_{n}$ are c-unified)

Unity $2 c r$ For any $x_{1}, \ldots, x_{n}, y$ (y c-unifies $x_{1}, \ldots, x_{n} \rightarrow y$ c-unifies $x_{1}, \ldots, x_{n}$ because $y, x_{1}, \ldots, x_{n}$ are r-unified)

Unity 2cc delivers a vicious regress: c-unification is explained by a c-unifier (by Unity 1Cc), which is explained by c-unification (by Unity 2cc), which is

15. A note on labelling. We will see a range of variations on Unity 1 and 2. Each connects one kind of unity to another that explains it. I have tried to label the principles in a way that makes this visible. Label endings are of the form ' $x y^{\prime}$, to indicate that $x$-unity is explained in terms of y-unity. Thus Unity Icc explains c-unity in terms of c-unity, and Unity 1 cr explains c-unity in terms of r-unity.

16. Strictly, Unity 1cc entails a version of (2cc) in which ' $g$ ' is replaced by a variable bound by an initial existential quantifier. The principle in the text follows from letting ' $g$ ' name an arbitrary witness to this generalisation. Likewise mutatis mutandis for (2cr) below.

17. An alternative option is: for any $x_{1}, \ldots, x_{n}, y$ (y c-unifies $x_{1}, \ldots, x_{n} \rightarrow y$ c-unifies $x_{1}, \ldots, x_{n}$ because $y$ r-unifies $\left.x_{1}, \ldots, x_{n}\right)$. This says that the composed thing is always a relation whose holding amongst the parts explains why they compose it. I think we can safely set such explanatory circularity aside. 
explained by a c-unifier (by Unity 1cc), which .... The explanation never leaves the compositional, hence fails to explain how compositionality is possible. So let us consider Unity $2 \mathrm{cr}$ instead.

Given (2cc), Unity 2cr entails:

(3cr) $g$ c-unifies $a, b, c, d$ because $g, a, b, c, d$ are r-unified.

Given the materials thus far, no regress follows. However, I see two problems with the resulting view.

Firstly, it is implausible that whenever some things compose another, they do so merely because they are related to it somehow or other. That is too undiscriminating a notion. Given an abundant conception of relations (Footnote 14), every plurality are related to every individual in many different ways, but no plurality compose every individual. This suggests that $x_{1}, \ldots, x_{n}$ being abundantly related to $y$ is too uninformative to really explain them composing $y$. Perhaps a sparse conception of relations will avoid this problem, though it's not entirely clear. For all I know, the following may be possible: (i) some plurality are sparsely related to something they compose, and (ii) some plurality are sparsely related to something they don't compose. If so, then this suggests that even $x_{1}, \ldots, x_{n}$ being sparsely related to $y$ is still too uninformative to explain them composing $y$. The view seems to need modifying so that what explains $x_{1}, \ldots, x_{n}$ composing $y$ is some particular way in which $x_{1}, \ldots, x_{n}$ are sparsely related to $y$, rather than just their being sparsely related somehow or other. ${ }^{18}$

Secondly, the view has potentially unattractive commitments. ${ }^{19}$ On this view, a plurality composes something because they compose a certain particular thing. And they compose that thing because of how they're (non-compositionally) related to it. So, for example, Tibbles's organs compose something because they compose Tibbles, because of how they're (non-compositionally) related to Tibbles. The

18. This is naturally implemented by replacing Unity $2 \mathrm{cr}$ with either of:

For any $x_{1}, \ldots, x_{n}, y\left(y\right.$ c-unifies $x_{1}, \ldots, x_{n} \rightarrow$ for some $z$ (y c-unifies $x_{1}, \ldots, x_{n}$ because $z$ r-unifies $\left.y, x_{1}, \ldots, x_{n}\right)$ )

For any $x_{1}, \ldots, x_{n}, y\left(y\right.$ c-unifies $x_{1}, \ldots, x_{n} \rightarrow \exists Z^{n+1}\left(y\right.$ c-unifies $x_{1}, \ldots, x_{n}$ because $\left.\left.Z^{n+1}\left(x_{1}, \ldots, x_{n}, y\right)\right)\right)$

The second problem in the main text afflicts these modified principles too. I discuss the second-order quantifier used in the second principle in Section 3 where I also propose a nearby alternative (Unity c) that avoids the problems in the main text.

19. My own view is that these commitments are unattractive only for cases of what Fine (2010 582) calls generative applications of compositional operations, i.e., when parts are metaphysically prior to whole. There is an attractive neo-Aristotelian tradition according to which the compositional operations connecting ordinary macroscopic substances, including ourselves, with their matter, are not of that kind. See, e.g., Wiggins (2001) or Jones (2015) for more. 
explanatory base thus includes both Tibbles and his organs. This places parts and whole on an even metaphysical footing, with both involved in explaining what composes what. There is no deep metaphysical sense in which the parts are prior to the whole on this view, or in which the unified whole emerges from the plurality of parts. The composition relation is explained, but not the existence of composed wholes. Many will find this implausible. And it does not explain how unities themselves are possible. So let us set this view aside.

We've just seen that Unity Icc does not deliver an adequate explanation of c-unification, whether combined with Unity $2 \mathrm{cc}$ or Unity $2 \mathrm{cr}$. So let us reject all three principles and consider Unity $1 \mathrm{cr}$ instead. That and (1) entail:

(2cr) $a, b, c, d$ are c-unified because $g$ r-unifies $a, b, c, d$.

We now have an explanation of c-unification in non-compositional terms: things compose (if they do) because a relation holds amongst them. The emergence of one from many is explained by relations holding amongst the many: relationality explains compositionality.

Does problematic regress arise? Not easily. The original regress was problematic because each instance of the target kind of unification (i.e., c-unification) was always eventually explained via another instance of that same kind of unification. To achieve that from Unity $1 \mathrm{cr}$ would require something to force an explanation of r-unification in terms of c-unification or c-unifiers. Either of the following would suffice:

Unity $2 r c$ For any $x_{1}, \ldots, x_{n}, y\left(y\right.$ r-unifies $x_{1}, \ldots, x_{n} \rightarrow y$ r-unifies $x_{1}, \ldots, x_{n}$ because $y, x_{1}, \ldots, x_{n}$ are c-unified)

Unity 3 For any $x_{1}, \ldots, x_{n}, y\left(y\right.$ r-unifies $x_{1}, \ldots, x_{n} \rightarrow y$ r-unifies $x_{1}, \ldots, x_{n}$ because $y$ c-unifies $\left.x_{1}, \ldots, x_{n}\right)$

We are, however, free to reject both principles, and to combine that with acceptance of Unity $1 \mathrm{cr}{ }^{20}$

Another way to obtain regress would be to explain relationality via relationality, as in: ${ }^{21}$

Unity $1 r r$ For any $x_{1}, \ldots, x_{n}\left(x_{1}, \ldots, x_{n}\right.$ are r-unified $\rightarrow$ for some $y\left(x_{1}, \ldots, x_{n}\right.$ are r-unified because $y$ r-unifies $\left.x_{1}, \ldots, x_{n}\right)$ )

20. An instance of Unity 2rc might be David Armstrong's (1996) theory of states of affairs or Jason Turner's (2016) factalism. On these views (roughly), objects and properties are derivative abstractions from states of affairs that combine them, and objects possess properties in virtue of their composing states of affairs with those properties. Unity 3 says that relations that hold amongst things are, and do so because they are, composed by those things. I do not know of any extant instances of this strange view.

21. I discuss the regress arising from Unity 1 rr further in Section 3 
Unity $2 r r$ For any $x_{1}, \ldots, x_{n}, y\left(y\right.$ r-unifies $x_{1}, \ldots, x_{n} \rightarrow y$ r-unifies $x_{1}, \ldots, x_{n}$ because $y, x_{1}, \ldots, x_{n}$ are r-unified)

Notice that Unity 2rr generates regress only in conjunction with Untiy 1rr, whereas Unity 1 rr itself suffices for regress. Unity 1 rr suffices for regress because $y$ holding amongst $x_{1}, \ldots, x_{n}$ is one way of $y, x_{1}, \ldots, x_{n}$ being related to one another, that is, being r-unified. This instance of $r$-unification can then be plugged back into the antecedent of Unity Irr to yield another r-unifier and hence instance of r-unification. And so on ad infinitum. ${ }^{22}$

Unity Irr captures the core of the Bradleyan regress of relations. Four points follow about this regress.

Firstly, even Priest employs relationality in his dialetheic account of unity. On his view, gluons unify by being identical to each of the things they unify. So, for example, gluon $g$ unifies $a, b, c, d$ because:

$$
g=a \wedge g=b \wedge g=c \wedge g=d
$$

The identity relation is clearly essential to this account. ${ }^{23}$

Secondly, this regress concerns relationality, not compositionality. Any problems that arise primarily concern relationality, not compositionality. Although such problems do concern compositionality indirectly-if compositionality is explained via relationality, as in Unity $1 \mathrm{cr}$ - they are not distinctive of compositionality. Any other phenomenon explained in relational terms is similarly afflicted. If Priest's argument that gluons cannot unify depends on this regress, it is equally an argument for the inadequacy of any relational explanation of anything and reveals nothing distinctive about compositionality.

22. One could block this regress by adopting a sparse conception of r-unification (see Footnote 14 and denying that $y$ holding amongst $x_{1}, \ldots, x_{n}$ involves $y, x_{1}, \ldots, x_{n}$ being sparsely related to one another. Although this is an option, advocates of Unity irr should resist it. It entails that the key theoretical primitive of the theory of relations, namely 'holds amongst', the relational analogue of 'instantiates', expresses an unnatural and gerrymandered notion. But according to Unity 1rr, relations do genuine explanatory work within metaphysics: when things are sparsely related, it's because a relation holds amongst them. Highly natural notions, like being sparsely related, shouldn't be explained in terms of much less natural ones. So the regress should not be blocked in this way. For discussion of the role of 'holds amongst' in supplying the theory of relations with content, see Jones 2017 Sections 2-3).

23. Perhaps Priest could respond by denying that identity is a relation, on the grounds that it never holds between distinct things. I see three difficulties with this suggestion. (i) Relations with reflexive instances are presumably unproblematic, e.g., being the same height as. But then it is unclear why the limiting case of relations like identity with only reflexive instances should be thought problematic. (ii) Identity is the negation of distinctness, a relation which unproblematically holds between (all and only) distinct things. Assuming that the negation of a relation is a relation, identity is a relation too. (iii) Even if identity isn't a relation, Priest's proposal still makes essential use of the conjunction relation expressed by ' $\wedge$ ', on which see Footnote 26 . 
Thirdly, the regress shows that one cannot explain how relationality is possible by invoking relations. Although each instance of relationality is explained given Unity $1 \mathrm{rr}$, it's explained by another instance of relationality having the form: $r$ holds amongst $x_{1}, \ldots, x_{n}{ }^{24}$ Relationality itself therefore goes unexplained. Is this problematic? It depends on our explanatory goals. If our goal is just to explain how compositionality is possible, however, then it is unproblematic. As was argued above, relationality and compositionality are distinct phenomena. So we may legitimately use relationality to explain compositionality, even if we cannot explain in other terms how relationality itself is possible. Every explanation must treat something as primitive; provided it's not what one seeks to explain, this is no threat to explanatory adequacy. One form of unity may thus be used to explain how a very different one is possible, as in Unity $1 \mathrm{cr}$.

Fourthly, it appears to be impossible in principle to explain how relationality itself is possible. So Unity icr cannot be faulted for using unexplained relationality to explain compositionality. The impossibility arises because an explanation of how relationality is possible is extremely close to an explanation of how predication is possible. But an explanation of how predication is possible requires an account of predication couched in predication-free terms, which is known to be impossible; I return to this at the end of Section 4 . The only way to explain relationality without explaining all predication is to somehow explain relationality in purely monadic terms. Yet it is doubtful whether this is possible, for the following reason.

It is hard to see how any such account could avoid using two-place sentential connectives like 'and' to connect monadic predications of different relata. For example, suppose one wants to use the distribution of monadic heights across individuals to explain the is taller than relation. What accounts for $a$ being taller than $b$, isn't merely that $a$ is six feet tall, say; and it isn't merely that $b$ is five feet tall, say. For each of those monadic height-predications is individually compatible with $a$ not being taller than $b$. Rather, what accounts for $a$ being taller than $b$ is this: $a$ is six feet tall and $b$ is five feet tall. ${ }^{25}$ Some sentential connective is needed

24. One could try to use relationality to explain how relationality is possible by restricting Unity 1 rr to exclude 'holds amongst' predications. Two points in response. (i) Although this blocks the regress, it leaves the relationality of holding amongst unexplained. The proposal therefore does not explain how relationality itself is possible, but merely uses one kind of relationality alongside a plenitude of relations to explain all others. (ii) The approach embodies an unattractive double standard. It treats the key theoretical primitive of the theory of relationsi.e., the 'holds amongst' relational predicate-differently from all other relational predicates used for serious theoretical work. It is hard to see what could motivate using relations to explain some theoretically important cases of relationality, but not those concerning what holds amongst what.

25. This argument generalises to any relation $R$ that lacks a base of monadic properties $F_{1}, F_{2}, \ldots$ such that (i) for each $F_{i}$, at least one of $F_{i}(x)$ and $F_{i}(y)$ necessitates $R(x, y)$, and (ii) $R(x, y)$ necessitates the disjunction whose disjuncts are $\left(F_{i}(x) \vee F_{i}(y)\right)$ for all the $F_{i}$. Most 
here to link a monadic predication of one relatum with a monadic predication of the other relatum, in order to obtain a distribution of monadic properties across both relata. Yet sentential connectives express relations on the semantic values of sentences. ${ }^{26}$ Any supposedly monadic account of relationality will therefore employ relationality after all, in the guise of logical relations like conjunction. If so, then it is impossible to explain relationality in purely monadic terms, and hence impossible to explain relationality in purely non-relational terms too. ${ }^{27} \mathrm{We}$ ought therefore to reject Unity $1 \mathrm{rr}$, and the project of explaining how relationality is possible along with it. ${ }^{28}$ Unity $1 \mathrm{cr}$ then provides a non-regressive explanation of how compositionality is possible.

On this combination of views, composition is always explained by relations holding amongst things (by Unity $1 \mathrm{cr}$ ), though relational truths and facts are not always explained in that way (since we reject Unity 1rr). This suggests that we could cut out the intermediary relations, and use relational facts (as opposed to relations holding amongst things) to explain composition directly instead, thereby avoiding any lingering concerns about Unity irr. The next section develops this kind of view.

The lesson, I think, is as follows. Priest presents his argument as a version

interesting relations appear to be like that. But if (i) and (ii) are satisfied, each instance $R(x, y)$ is necessitated by a monadic predication $F_{i}(x)$ or $F_{i}(y)$, at least opening the possibility of reducing $R$ to such predications.

26. One could respond by denying that the connectives express relations or have worldly correlates of any kind; they belong instead to our primitive theoretical ideology. This is too big an issue to address properly here. I will merely sketch why I am not attracted to this approach, without pretending to settle the matter. Firstly, non-denotational treatments of the connectives make it difficult to formulate a generalised semantic theory-I believe this terminology is due to Linnebo and Rayo (2012 275) - in which one can generalise about all the different meanings any expression could in principle have had, including the connectives. By contrast, denotational treatments of the connectives extend smoothly into generalised semantic theories by enabling generalisation over the denotations of the connectives. But these connective-denotations are presumably relations, or something very much like them. Secondly, I am attracted to systems of higher-order logic that permit quantifiers to bind variables of all semantic types, including that of the connectives. A suitable notion of identity and the validity of existential generalisation will then deliver theorems like ' $\exists X(X=\wedge)^{\prime}$, where ' $X$ ' is a variable of the same type as binary sentential connectives, and ' $=$ ' is an identity predicate taking binary connectives in both argument positions. We can think of that formula as saying that there (higher-order) is a conjunction relation. I return to higher-order quantification and identity in Section 3 and Section 4 On higher-order identity, see also Dorr (2016).

27. MacBride (2016) discusses several other problems for monadic analyses of relationality.

28. This does not entail that we should reject the existence of relations. One may believe that relations exist because one takes them to do theoretical work that does not require Unity Irr's truth. For example, relations allow us to formulate first-order generalisations encoding patterns of relational commonality that would otherwise require higher-order quantification, and to provide referents for predicate-nominalisations like 'wisdom'. See Section 3 and Footnote 32 for more. 
of Bradley's regress. Bradley's goal was to show that relationality is incoherent, and his regress argument specifically concerns relationality, not compositionality. Although the Bradleyan regress does not show that relationality is incoherent, ${ }^{29}$ it does show that one cannot explain relationality itself by invoking relations; for relational 'holds amongst'-predications are required by any such account. The underlying mistake driving Priest's argument is to assimilate compositionality too closely to relationality, and to treat the Bradleyan argument's conclusion as concerning compositionality as a result. Once these notions are distinguished, however, Bradleyan regress is no obstacle to an adequate relational account of compositionality.

Priest presents two objections to views that appeal to relations. Here's the first:

There is already a confusion at the heart of this thought ... between relating and unifying. Relations do not, in general, unify. I am related to my mother by bearing the relationship of child to her. ... But obviously the relation does not serve to render my mother and myself a unity in the appropriate sense. (12)

However, Unity 1cr does not entail that relations always c-unify. It says that each case of compositionality is explained by some case of relationality, not that each case of relationality explains some case of compositionality. The proposal requires a distinction between relations whose relating c-unifies their relata, and those whose relating does not. But I see no obvious reason to regard that as problematic.

Problems will arise if this distinction can only be explained in compositional terms, as, for example, the distinction between the relations whose holding explains composition and those whose holding does not. I cannot settle here whether that is so. But other options are available. Perhaps the distinction is utterly simple and fundamental. Or perhaps the unifying relations are those whose holding suffices for a life in Peter van Inwagen's (1990) sense, or for consciousness à la Trenton Merricks (2003). The framework of Kit Fine's (2010) provides an alternative model, on which the distinction will depend on the kind of whole and mode of composition at issue. It will be difficult to articulate the distinction precisely for non-toy examples of actual wholes. But that is a difficulty of practice not principle, and is just what we should expect of our messy and complex world.

Here's Priest's second objection to invoking relations:

29. Given that the Bradleyan regress arises primarily from Unity $1 \mathrm{rr}$, it shows that relationality is incoherent only if both (i) the coherence of relationality requires the truth of Unity $1 \mathrm{rr}$, and (ii) the explanatory regress arising from Unity $1 \mathrm{rr}$ shows that it is false. Whatever one thinks about (ii), one should reject (i). Nominalists about relations reject (i), as may those whose reasons for believing in relations do not require Unity 1 rr (see Footnote 28). Perhaps those views are false, but they're certainly not incoherent. 
When one invokes the [relation] in question, one simply adds an extra element to the melange. If one is puzzled by unity in the first case, one should be equally puzzled by the supposed unity in the second.... We have the parts plus the relationship between them. ... How is this any better? (12-13)

However, when relations occur in Unity 1cr, it is not merely as an "element of the melange". The explanation of c-unification does not merely exhibit a relation alongside the parts but says, of relation and parts, how they are: the relation holds amongst the parts. From a logico-semantic perspective, the explanation is expressed not by a singular term for the relation alone, or even by a plural term for relation and parts. The explanation is expressed by a whole sentence which says, of the relation and parts, how they are configured. Following David Lewis 1992 206-207), we should distinguish two fundamentally different matters: what there is and how things are. The latter does not reduce to the former. ${ }^{30}$ In the quote above, Priest seems to assume that an appeal to relations is an appeal merely to what there is, whereas Unity Icr's appeal to relations is an appeal to how things are. This explanation does not merely exhibit a unifying individual, but says how it stands to other individuals. So puzzlement about compositionality need not, and should not, extend to the relationality that explains it. In short, gluons glue and thereby give rise to (c-)unity by being relations that hold amongst the parts of composed wholes.

\section{How to Unify Without Things that are Gluons}

We've seen how gluons can unify, even if they are things. That suffices to show that compositionality is not inherently contradictory. The second horn of Priest's dilemma maintains that unity cannot arise if gluons are not things-that is, if there are no such things as gluons-because then nothing unifies the parts of

30. Note that these matters are not independent. For example, if a relation holds amongst things (how things are), then there is that relation (what there is). How things are does not reduce to what there is because the latter underdetermines the former: the relation's existence underdetermines what it holds amongst. For an attempt to overcome this obstacle, see Armstrong's (1989) and (1996) theory of truthmakers and states of affairs. Armstrong joins typical theories of relations in accepting: when $R(a, b)$, there is a relation $R$ and individuals $a$ and $b$, whose joint existence is (typically) compatible with $\neg R(a, b)$, and such that $R$ holds amongst $a$ and $b$ (which necessitates $R(a, b)$ ). Armstrong goes beyond typical theories of relations in also accepting: when $R(a, b)$, there is an individual that somehow combines $R$ with $a$ and $b$, and whose mere existence is incompatible with $\neg R(a, b)$. Armstrong calls these individuals states of affairs. He attempts to reduce how things are to what there is by using the existence of states of affairs to account for all of how things are. Note, however, that the existence of states of affairs, and hence the availability of this reduction, is not entailed by realism about relations. See Lewis 1992 for criticism of Armstrong's approach. 
unities. I now generalise the previous section's proposal to show that this is not so.

Central to my proposal is:

Unity 1 cr For any $x_{1}, \ldots, x_{n}\left(x_{1}, \ldots, x_{n}\right.$ are c-unified $\rightarrow$ for some $y\left(x_{1}, \ldots, x_{n}\right.$ are c-unified because $y$ r-unifies $\left.x_{1}, \ldots, x_{n}\right)$ )

The consequent existentially quantifies over r-unifiers. To r-unify some things is to be a relation that holds amongst them. So the proposal requires realism about relations, the thesis that there are such things as relations. The proposal is therefore incompatible with nominalism about relations, the thesis that there are no such things as relations. I will now show how the proposal can be reconstrued in nominalistically acceptable terms, to yield an account of unity without relations (a.k.a. gluons).

Consider ordinary cases of relationality first, not those involved in c-unification. Realists accept claims like:

Being on holds amongst Tibbles and the mat (in that order).

Being between holds amongst Birmingham, Oxford, and Sheffield (in that order).

Because those claims mention relations, nominalists reject them in favour of claims like:

Tibbles is on the mat.

Birmingham is between Oxford and Sheffield.

Where realists have a property-name and variably polyadic 'holds amongst' predicate, nominalists have only familiar relational predications. Now, consider the particular explanations of c-unification provided by Unity $1 \mathrm{cr}$. Those are claims like: ${ }^{31}$

$a_{1}, \ldots, a_{n}$ compose something because being arranged cat-wise holds amongst $a_{1}, \ldots, a_{n}$.

$b_{1}, \ldots, b_{n}$ compose something because being arranged table-wise holds amongst $b_{1}, \ldots, b_{n}$.

Nominalists can achieve the same explanatory effect without mentioning relations by instead employing claims like:

$a_{1}, \ldots, a_{n}$ compose something because $a_{1}, \ldots, a_{n}$ are arranged cat-wise. order.

31. For simplicity, I elide the distinction between relations and plural properties, and ignore 
$b_{1}, \ldots, b_{n}$ compose something because $b_{1}, \ldots, b_{n}$ are arranged table-wise.

The explanatory power of each instance of Unity Icr can thereby be achieved without mention of relations or use of the 'holds amongst' predicate characteristic of realism. One can thus use relationality to explain each instance of compositionality via nominalistically acceptable means, and without invoking such things as relations (a.k.a. gluons). The result is a nominalistically acceptable alternative to each specific explanation of c-unification provided by Unity $1 \mathrm{cr}$.

However, we do not yet have a nominalistically acceptable alternative to Unity Icr itself. Systematic theory, as opposed to a mere collection of theses, requires a single generalisation from which these specific explanations follow, a principle that captures the common pattern they exhibit. Yet it is difficult to formulate such a principle. ${ }^{32}$ I now consider three variants of Unity $1 \mathrm{cr}$ one might invoke to do this job.

As a first pass, we might try:

Unity $a$ For any $x_{1}, \ldots, x_{n}\left(x_{1}, \ldots, x_{n}\right.$ are c-unified $\rightarrow x_{1}, \ldots, x_{n}$ are cunified because $x_{1}, \ldots, x_{n}$ are r-unified)

However, r-unification requires only that $x_{1}, \ldots, x_{n}$ stand in some relation or other (as the realist would put it). But things that compose will typically stand in many different relations, only some of which are relevant to whether (and what) they compose. Unity a does not capture this and is therefore inadequate.

A natural response is to invoke ways of being related, as in:

Unity $b$ For any $x_{1}, \ldots, x_{n}\left(x_{1}, \ldots, x_{n}\right.$ are c-unified $\rightarrow$ for some way of being related $\left(x_{1}, \ldots, x_{n}\right.$ are c-unified because $x_{1}, \ldots, x_{n}$ are related in that way))

What are these ways of being related? They seem very much like relations. Unity $\mathrm{b}$ then collapses into the original, nominalistically unacceptable, Unity $1 \mathrm{cr}$.

The solution is to employ a different kind of generalisation in the consequent of Unity 1cr. That existential quantifier is first-order: it binds variables in name position. But there are also higher-order quantifiers that bind variables of other semantic types. In particular, second-order quantifiers bind variables in predicate position. We can use second-order quantifiers to formulate the following variant of Unity $1 \mathrm{cr}: 33$

Unity $c$ For any $x_{1}, \ldots, x_{n}\left(x_{1}, \ldots, x_{n}\right.$ are c-unified $\rightarrow \exists Y^{n}\left(x_{1}, \ldots, x_{n}\right.$ are c-unified because $\left.Y^{n}\left(x_{1}, \ldots, x_{n}\right)\right)$ )

32. This is an instance of a familiar problem for nominalism. See Devitt (1980), Armstrong (1980), and Lewis (1983 esp. 24-25) for more.

33. See van Cleve (1994) for a similar application of second-order quantification, reacting to the Devitt-Armstrong exchange about "ostrich nominalism" cited in Footnote 32 
This captures all the particular nominalist alternatives to the explanations deriving from Unity $1 \mathrm{cr}$. If second-order generalisation is compatible with nominalism, then relationality can explain compositionality without things that are gluons. ${ }^{34}$

Is second-order quantification compatible with nominalism? It depends what second-order quantifiers mean. I will not attempt to settle this here. I will instead simply contrast two views and state my preference for the second.

According to the first view, second-order quantifiers range over a special kind of thing, such as sets, extensions, intensions, or properties and relations. These can be referred to with names of the metalanguage, and are in the domain of its first-order quantifiers. This is the kind of semantics employed in standard model-theoretic analyses of second-order quantification. ${ }^{35}$ If this captures what second-order quantifiers mean, then Unity c is really just a notational variant of Unity $1 \mathrm{cr}$ (at least, as viewed from our metalinguistic perspective when we invoke these things to explain what second-order quantifiers mean).

According to the second view, second-order quantification is primitive: the meaning of second-order quantifiers can only be captured by using second-order quantifiers in the metalanguage (whose meaning can only be captured by using second-order quantifiers in a meta-metalanguage, ...). On this view, secondorder quantification is a sui generis form of non-objectual and non-substitutional quantification. As a result, Unity c is not semantically equivalent to Unity $1 \mathrm{cr}$ and is not committed to there being things that are relations. Its ontological commitments are just those already implicit in each of its instantiations, that is, the nominalistically acceptable explanations of compositional unity outlined above. ${ }^{36}$

Primitivism about second-order quantification is controversial. Although plausibly present in natural languages like English, the case is not watertight. ${ }^{37}$ So for argument's sake, let us be concessive and grant primitivism's opponents that second-order quantification isn't present in English. Then one cannot use one's prior understanding of second-order quantification in English to say what the second-order formalism means. When one attempts to say in English what

34. Here are two nearby alternatives to Unity c. (1) Schematise the nominalist explanations of c-unification and assert that some instance holds for each c-unified $x_{1}, \ldots, x_{n}$. However, this cannot accommodate c-unification explained by relations in principle inexpressible in any possible language. The possibility of such relations is part and parcel of an adequately full-blooded realism, according to which reality is entirely independent of, and hence not at all constrained or restricted by, our linguistic abilities. (2) Employ quantification into sentence position in place of second-order quantification, thus: for any $x_{1}, \ldots, x_{n}\left(x_{1}, \ldots, x_{n}\right.$ are c-unified $\rightarrow \exists p\left(x_{1}, \ldots, x_{n}\right.$ are c-unified because $\left.\left.p\right)\right)$. I focus on Unity c because it is more informative: Unity c entails this proposal but not conversely.

35. See Shapiro (1991) for a thorough discussion.

36. Hale (2013 $180-189)$ develops this idea in response to Quine's 1986 68) famous criticism of second-order logic as "set theory in sheep's clothing".

37. See Rayo and Yablo 2001 for the positive case and references. 
it means, one is forced to use a first-order gloss, which conflicts with the primitivist interpretation. So in order to come to understand primitivist second-order quantification, one must take a different approach. The most prominent option is what Williamson (2003 459) calls the direct method of immersion into a practice of using a second-order language. Even if one doesn't yet understand primitive second-order quantification, I see no obstacle to coming to understand it in this way.

Why take primitivism seriously? Why think that there really is a primitivist interpretation out there for us to grasp? The answer has two parts. Firstly, because there are, as far as I'm aware, no compelling arguments against primitivism. ${ }^{38}$ Moreover, many now accept a primitivist interpretation of at least one kind of non-first-order quantification, namely plural quantification. Yet once the intelligibility of one form of primitive non-first-order quantification is admitted, a central obstacle to admitting more is removed. Secondly, primitive secondorder quantification has proven to be theoretically useful, with applications in metaphysics, as well as the philosophy of logic, language, and mathematics. ${ }^{39}$ Although these applications do not establish that a primitivist interpretation is available, they should suffice for regarding it as a live theoretical option. For those unconvinced by primitivism, however, I'd like to emphasise that Section 2 's account of unity does not require it; only this section's nominalist alternative to Unity $1 \mathrm{cr}$ requires primitive second-order quantification. I assume a primitive interpretation of all higher-order quantifiers henceforth. $4^{\circ}$

Primitivist interpretations of second-order quantification sever the connection between quantification and thinghood driving the second horn of Priest's dilemma. The problem was that if gluons are not things, then no plurality is unified because

38. For discussion and references, see Prior 1971 Chapter 3), Boolos 1975), Hale 2013 180-189), and Turner (2015).

39. For applications in logic, language, and mathematics, see, e.g., Prior (1971 Chapter 3), Wright (1983), Boolos (1985), Williamson (2003), Wright (2007), Linnebo and Rayo (2012), Jones (2016), and Bacon and Russell (2017). For applications within metaphysics, see, e.g., van Cleve (1994), Dunaway (2013), Williamson (2013 254-261), Dorr (2016), Jones (2017), and Goodman (2017).

40. Primitive second-order quantification also enables a non-regressive explanation of relations holding amongst things. We can explain relations holding amongst things via particular ways of them being related:

For any $n$-adic relation $y$, there is $Y^{n}$ such that, for any $x_{1}, \ldots, x_{n}\left(y\right.$ r-unifies $x_{1}, \ldots, x_{n} \rightarrow y$ r-unifies $x_{1}, \ldots, x_{n}$ because $\left.Y^{n}\left(x_{1}, \ldots, x_{n}\right)\right)$.

For example, being between holds amongst Birmingham, Sheffield, and Oxford because Birmingham is between Sheffield and Oxford. This approach uses primitive relationality to explain relations holding amongst things. It avoids the Bradleyan regress arising from Unity 1 rr because $Y^{n}\left(x_{1}, \ldots, x_{n}\right)$ involves $x_{1}, \ldots, x_{n}$ being related but not a thing that is a relation holding amongst them. 
no thing unifies them. Yet according to primitivism, the truth of a second-order existential generalisation does not require there to be a thing that witnesses the generalisation. For 'thing' is a first-order notion, applicable to (all and only) values of first-order variables and potential referents of names. This allows for a plurality that is unified because there (second-order) is a unifier supplied by Unity c, even though no (first-order) thing unifies them..$^{41}$

We now have three accounts of unity, each with its own costs and benefits:

(A) Section 2 2 s account based around Unity $1 \mathrm{cr}$, with its ontology of relations and 'holds amongst' predicate.

(B) This section's account based around Unity c, which dispenses with relations in favour of primitive second-order quantification.

(C) Priest's gluon theory, which requires true contradictions and failures of modus ponens and the transitivity of identity. ${ }^{42}$

Those who value consistency, the transitivity of identity, and Leibniz's Law should go for (A) or (B). Nominalists about relations should go for (B) or (C). And opponents of primitive higher-order quantification should go for (A) or (C). My own view is that (B) is preferable, since I think I already understand primitive higher-order quantification and so see no cost in appealing to it here. Whether or not I am right to prefer (B), however, it is clear that neither horn of Priest's dilemma shows that unity is contradictory; at least, not without non-trivial commitments elsewhere. For (A) shows that gluons can consistently unify, even if they are things, while (B) shows that consistent unification does not require things that are gluons.

\section{Revenge?}

Revenge problems arise when the theoretical resources used to resolve a problem allow us to formulate a new instance of the problem that cannot be resolved by applying those resources in the same way. The classic example is the liar paradox.

41. Priest also claims that unifiers must be things because we can speak and think about them. A similar response is available. To speak of non-thing gluons is to use predicates $R$ appearing in true claims of the form: $a_{1}, \ldots, a_{n}$ are c-unified because $R\left(x_{1}, \ldots, x_{n}\right)$. To think of non-thing gluons is to think thoughts whose contents are expressible using such predicates. Given a nominalistically acceptable conception of predication, this does not require things that are gluons.

42. True contradictions because Priest-style gluons both are and are not things. Nontransitive identity because Priest-style gluons unify pluralities by being identical to each of their elements. Failures of modus ponens because transitivity is derivable from Leibniz's Law ( $a=b$ iff $\forall X(X a \leftrightarrow X b))$, universal instantiation, and modus ponens, the first two of which hold in Priest's system. 
The liar sentence 'this sentence is false' is true iff it is false, yet truth and falsity are exclusive and exhaustive. One standard response says that the original liar sentence is neither true nor false, but has a special intermediate alethic status. We can use this intermediate status to formulate a new strengthened liar sentence, 'this sentence is false or intermediate'. This is just as paradoxical as the original sentence: if it's true then it's false or intermediate, if it's false then it's true, and if it's intermediate then it's true, yet truth, falsity, and intermediacy are supposed to be exclusive and exhaustive. ${ }^{43}$

This section considers a revenge problem for Section 3 s proposal, and uses it to argue that a version of the unity of propositions is (one of) reality's most fundamental forms of unity. To be clear, however, the problem is not specific to this proposal. The problem arises for any view that permits higher-order quantification. It specifically afflicts Section 3 s proposal only because Unity c employs higher-order quantification. But neither Section $2 \mathrm{l}$ s proposal nor Priest's proposal is incompatible with higher-order quantification. So if higher-order quantification is in fact legitimate, and if the revenge problem is sound, then all three proposals fail; whereas, if higher-order quantification is illegitimate, then Priest's proposal and Section 2 2 s proposal are both available. I explain how the problem arises for Priest's proposal below, though I focus primarily on Unity c.

The goal is to explain how one emerges from many. According to Unity c, each case of compositionality is explained by some case of relationality. This proposal is adequate only if relationality is not a form of the emergence of one from many. Otherwise, vicious regress arises; for each case of compositionality is then explained by some case of compositionality, thereby leaving compositionality itself unexplained. Unity c makes essential use of higher-order quantification. Yet the apparatus of higher-order logic seems to provide a sense in which relationality is a form of the emergence of one from many. I now explain why.

Given higher-order quantification into predicate position, it is natural to permit quantification into other positions too. For present purposes, the key examples are sentence position, and sentential operator position. One can use these to characterise an identity relation on the type of sentences: ${ }^{44}$

$$
\forall p \forall q(p=q \leftrightarrow \forall \phi(\phi(p) \leftrightarrow \phi(q)))
$$

We can use this sentential identity relation to express a sense in which that which explains c-unification is a one arising from many.

Suppose that $a, b$ are c-unified. Then by Unity c, for some $Y^{2}: a, b$ are c-unfied because $Y^{2}(a, b)$. Let the dyadic relational predicate ' $R$ ' express this $Y^{2}$. Then $a, b$

43. Another notable revenge problem concerns type-theoretic accounts of absolute generality. See Williamson 2003 esp. 458-459), Krämer (2017), and Florio and Jones (2018) for discussion.

44. ' $p$ ' and ' $q$ ' are sentential variables. ' $\phi$ ' is a one-place sentential operator variable. 
compose something because:

$$
R(a, b)
$$

Now, by the characterisation of sentential identity above, we have:

$$
R(a, b)=R(a, b)
$$

Given standard rules for the quantifiers, that entails:

$$
\exists p(R(a, b)=p)
$$

This provides a higher-order sense in which that which explains c-unification is a one: it is identical to something (though according to primitivism about higher-order quantification, this use of 'thing' is a misleading artefact of the English gloss). There is also a sense in which it combines many:

$$
\exists X^{2} \exists y \exists z\left(R(a, b)=X^{2}(y, z)\right)
$$

The elements of this many are drawn from different types, as is the one which they form. But one arises from many nonetheless: predicational and objectual aspects of reality combine into sentential, or propositional, aspects of reality. ${ }^{45} \mathrm{In}$ this sense, each case of relationality is also a case of cross-type compositionality. The revenge problem can now be stated: according to Unity c, each case of compositionality is explained by a case of cross-type compositionality; those explanations therefore presuppose compositionality and so do not explain how it is possible.

Call this kind of unity predicationality. Thinking in first-order terms, predicationality is the unity of propositions. According to primitivism about higher-order quantification, however, this gloss is misleading because it concerns things that are propositions. Given primitivism, predicationality can be accurately described and understood only by speaking and thinking in higher-order terms.

The account of compositionality in Section 3 employs predicationality. We have just seen a sense in which predicationality is a form of compositionality. ${ }^{46}$ It appears to follow that Section 3 s account of compositionality uses a form of

45. For more on this type-theoretic conception of reality itself, as opposed to merely the language used to describe it, see Williamson (2013 260-261), and Florio and Linnebo 2016 Section 7), Jones (2016), Jones (2017), and Florio and Jones (2018).

46. In the interests of simplicity, I gloss over the following subtlety in the text. Predicationality does not directly explain compositionality. Predicational unification of $R, a, b$ requires only that some $p$ predicationally unifies them, not that it be the case that $p$. To see why, note that this $p^{\prime}$ s existence-i.e., $\exists p(p=R(a, b))$-follows from $R(a, b)=R(a, b)$. Yet that follows from the characterisation of sentential identity alone and does not entail that $R(a, b)$. According to Unity c, $a, b$ are c-unified because $R(a, b)$. So that which explains c-unification is not merely predicational unification of $R, a, b$; it's the strictly stronger claim that $R(a, b)$. In first-order terms, predicationality is the kind of unity exhibited by propositions, not the kind of unity that 
compositionality to explain compositionality, and hence does not explain how compositionality is possible. How problematic is this? I now provide three reasons not to regard it as problematic.

Firstly, and least concessively, one can deny that predicationality is a form of compositionality; the appearance otherwise is an illusion generated by ignoring the type distinctions at the heart of higher-order logic. For example, standard systems of higher-order logic do not permit argument positions that accept expressions of more than one type. So the ordinary identity predicate and identity relation are distinct from the sentential and predicational notions characterised above. And the ordinary composition predicate and composition relation are distinct from the predicational notions currently at issue. On this view, it is really just equivocation to use the same notation for each, or to regard them as instances of a single phenomenon.

Although I am sympathetic to this first response, it requires controversial assumptions about the structure of higher-order logic. Systems of cumulative type theory have been developed that relax these assumptions. ${ }^{47}$ This makes conceptual space for a sense in which compositionality and predicationality are instances of a single phenomenon. My second and third responses to the revenge problem are more concessive, and require no such controversial assumptions.

Secondly, predicationality is an intrinsically cross-type form of unity. It combines the incommensurably different aspects of reality expressed by, for example, singular terms and predicates into those expressed by sentences. The kind of compositionality with which we began, however, is an intra-type notion: it combines pluralities of things into further things, remaining within the first-order domain. The instantiations of Unity c explain intra-type compositionality using inter-type predicationality. So even if predicationality is a form of compositionality, my proposal explains one kind of instance in terms of another very different kind. On this view, inter-type predicationality is the fundamental form of compositionality and explains all others. In particular, the familiar cases of intra-type compositionality with which we began are explained in inter-type terms.

Thirdly, one cannot avoid taking predicationality as primitive. Every theory, and every explanation, employs whole sentences composed out of other elements. But predicationality is the kind of unity that combines those elements' worldly correlates into propositional wholes. So every theory and every explanation presupposes predicationality. As Lewis put it:

accounts for their truth or the existence of facts. An instance of the latter kind of unity, not predicationality, is what directly explains c-unification. The revenge problem is that the $p$-i.e., $R(a, b)$-whose being such that $p$ explains c-unification of $a, b$ is itself a predicational unity: $a, b$ are c-unified because a certain predicational unity is a $p$ such that $p$, namely $R(a, b)$. That's the sense in which Unity c presupposes predicationality.

47. For recent discussion, see Degen and Johanssen (2000), Linnebo and Rayo (2012), Krämer (2017), and Florio and Jones (2018). 
Doing away with all unanalysed predication is an unattainable aim, and so an unreasonable aim. No theory is to be faulted for failing to achieve it. For how could there be a theory that names entities, or quantifies over them, in the course of its sentences, and yet altogether avoids primitive predication? Artificial tricks aside, the thing cannot be done. (Lewis 1983

Lewis's notion of predication here is not quite our own. His concern is with true predication because he's responding to Armstrong's (1978; 1980) "one over many" argument for realism about universals, and Armstrong's universals are intended to explain true predication. Our concern, however, is with the meaningfulness of predication. ${ }^{4}$ But the point is essentially the same. Every theory presupposes, hence does not explain, predicationality.

Here's how Priest's proposal employs predicationality. He proposes explaining unity via identity between each of the many parts and a single gluon. So where $g$ is the gluon of $a, b$, they are unified because:

$$
a=g \wedge b=g
$$

That is a unity because it is identical to something:

$$
\exists p(p=(a=g \wedge b=g))
$$

And it also combines many:49

$$
\exists \phi^{2} \exists X^{2} \exists x \exists y \exists z\left((a=g \wedge b=g)=\phi^{2}\left(X^{2}(x, z), X^{2}(y, z)\right)\right)
$$

So even Priest uses predicationally unified wholes to explain compositionality.

It is in principle impossible to explain predicationality in other terms. In this sense, predicationality is one of reality's two most fundamental forms of unity. The other is the higher-order analogue of the unity of facts, that is, the unity characteristic of being a $p$ such that $p .5^{50}$ Call this notion being the case. Predicational unity combines $R, a, b$ into a propositional whole $R(a, b)$. But it doesn't unify them sufficiently to determine whether or not $R(a, b)$. The additional unification supplied by being the case is required for that. Like predicational unity, it is in principle impossible to explain being the case in other terms,

48. This is a misleading way to put it because our concern is not with language, but with what language says. Predicationality, and the more familiar topic of the unity of propositions, is the metaphysical analogue of the linguistic notion of meaningful predication.

49. $\phi^{2}$ is a variable in the position of a 2-place sentential operator: it combines with two sentences to form a sentence. Instantiating ' $\phi^{2 \prime}$ with ' $\wedge$ ', ' $X{ }^{2}$ ' with the ordinary identity predicate, ' $x$ ' with ' $a$ ', ' $y$ ' with ' $b$ ', and ' $z$ ' with ' $g$ 'yields the triviality: $(a=g \wedge b=g)=(a=$ $g \wedge b=g)$.

50. In lambda notation, ( $\lambda$ p.p). 
because it is employed by every explanation and theory whatsoever. These are reality's two most fundamental forms of unity, mutually supporting components of metaphysical bedrock.

In conclusion, the revenge problem fails because the predicational unity presupposed by Unity c (as well as by both Unity 1cr and Priest's gluon-theory) is absolutely fundamental and presupposed by any explanation of anything whatsoever. That is compatible with using predicationality to explain other kinds of unity, including the (intra-type) compositionality with which we began. Section 2 showed how to do so, given realism about relations. And Section 3 showed how to do so without things that are relations. Either way, there is no obstacle to providing a consistent account of compositionality in other terms.

\section{Acknowledgements}

For comments, advice, and discussion, I'm grateful to Salvatore Florio, Chris Jay, Graham Priest, Henry Taylor, Rob Trueman, Al Wilson, and two anonymous referees, as well as audiences in York, Glasgow, Birmingham, and Cambridge This research was funded by an AHRC Leadership Fellowship.

\section{References}

Armstrong, David (1989). A Combinatorial Theory of Possibility. Cambridge University Press.

Armstrong, David (1996). A World of States of Affairs. Cambridge University Press. Armstrong, David (1978). Universals and Scientific Realism Volume 1: Nominalism and Realism. Cambridge University Press.

Armstrong, David (1980). Against "Ostrich Nominalism": A Reply to Michael Devitt. Pacific Philosophical Quarterly, 61(4), 440-449. Reprinted in Mellor and Oliver (1997: Chapter 9).

Bacon, Andrew and Jeffrey Sanford Russell (2017). The Logic of Opacity. Philosophy and Phenomenological Research. Advance online publication. https: //doi.org/10.1111/phpr.12454

Boolos, George (1975). On Second-Order Logic. Journal of Philosophy, 72(16), 509527. Reprinted in Boolos (1998: Chapter 3).

Boolos, George (1985). Nominalist Platonism. The Philosophical Review, 94(3), 327344. Reprinted in Boolos (1998. Chapter 5).

Boolos, George (1998). Logic, Logic and Logic. Harvard University Press.

Bradley, Francis H. (1897). Appearance and Reality, (second ed.). Clarendon Press. Degen, Wolfgang and Jan Johanssen (2000). Cumulative Higher-Order Logic as a Foundation for Set Theory. Mathematical Logic Quarterly, 46(2), 147-170. 
Devitt, Michael (1980). “Ostrich Nominalism" or “Mirage Realism"? Pacific Philosophical Quarterly, 61(4), 433-439. Reprinted in Mellor and Oliver (1997. Chapter 8).

Dorr, Cian (2016). To Be F Is to Be G. Philosophical Perspectives, 30, 39-134.

Dunaway, Billy (2013). Modal Quantification without Worlds. In Karen Bennett and Dean W. Zimmerman (Eds.), Oxford Studies in Metaphysics Volume 8 (151186). Oxford University Press.

Fine, Kit (2010). Towards a theory of part. Journal of Philosophy, 107(11), 559-589. Florio, Salvatore and Nicholas K. Jones (2018). Unrestricted Quantification and the Structure of Type Theory. Manuscript in preparation.

Florio, Salvatore and Øystein Linnebo (2016). On the Innocence and Determinacy of Plural Quantification. Nô̂s, 50(3), 565-583.

Goodman, Jeremy (2017). Reality Is Not Structured. Analysis, 77(1), 43-53.

Hale, Bob (2013). Necessary Beings: An Essay on Ontology, Modality, and the Relations Between Them. Oxford University Press.

Hawley, Katherine (2006). Principles of Composition and Criteria of Identity. Australasian Journal of Philosophy, 84(4), 481-493.

Jones, Nicholas K. (2015). Multiple Constitution. In Karen Bennett and Dean W. Zimmerman (Eds.), Oxford Studies in Metaphysics Volume 9 (217-261). Oxford University Press.

Jones, Nicholas K. (2016). A Higher-Order Solution to the Problem of the Concept Horse. Ergo: An Open Access Journal of Philosophy, 3(6), 1-35.

Jones, Nicholas K. (2017). Nominalist Realism. Noûs. Advance online publication. https://doi.org/10.1111/nous.12193

Krämer, Stephan (2017). Everything, and then Some. Mind, 126(502), 499-528.

Lewis, David (1983). New Work for a Theory of Universals. The Australasian Journal of Philosophy, 61(4), 343-77. Reprinted in Lewis (1999. Chapter 1).

Lewis, David (1992). Armstrong on Combinatorial Possibility. The Australasian Journal of Philosophy, 70(2), 211-224. Reprinted in Lewis (1999. Chapter 12).

Lewis, David (1999). Papers in Metaphysics and Epistemology. Cambridge University Press.

Linnebo, Øystein and Agustin Rayo (2012). Hierarchies Ontological and Ideological. Mind, 121(482), 269-308.

MacBride, Fraser (2016). Relations. In Edward N. Zalta (Ed.), The Stanford Encyclopedia of Philosophy (Winter 2016 ed.). Retrieved from https://plato. stanford.edu/archives/win2016/entries/relations/

Mellor, David H. and Alex Oliver (Eds.) (1997). Properties. Oxford University Press.

Merricks, Trenton (2003). Objects and Persons. Oxford University Press.

Perovic, Katarina (2017). Bradley's Regress. In Edward N. Zalta (Ed.), The Stanford Encyclopedia of Philosophy (Winter 2017 ed.). Retrieved from https: 
//plato.stanford.edu/archives/win2017/entries/bradley-regress/

Priest, Graham (2014). One: Being an Investigation into the Unity of Reality and of Its Parts, Including the Singular Object which is Nothingness. Oxford University Press.

Prior, Arthur N. (1971). Objects of Thought. Peter T. Geach and Anthony J. P. Kenny (Eds.). Oxford University Press.

Quine, Willard V. O. (1986). Philosophy of Logic, (second ed.). Harvard University Press.

Rayo, Agustin and Stephen Yablo (2001). Nominalism Through DeNominalization. Noûs, 35(1), 74-92.

Shapiro, Stewart (1991). Foundations Without Foundationalism: a Case for SecondOrder Logic. Oxford University Press.

Turner, Jason (2015). What's So Bad about Second-Order Logic? In Alessandro Torza (Ed.), Quantifiers, Quantifiers, and Quantifiers: Themes in Logic, Metaphysics, and Language (463-487), Vol. 373 of Synthese Library. Springer.

Turner, Jason (2016). The Facts in Logical Space. Oxford University Press. van Cleve, James (1994). Predication without Universals? A Fling with Ostrich

Nominalism. Philosophy and Phenomenological Research, 54(3), 577-590.

van Inwagen, Peter (1990). Material Beings. Cornell University Press.

Wiggins, David (2001). Sameness and Substance Renewed. Cambridge University Press.

Williamson, Timothy (2003). Everything. Philosophical Perspectives, 17(1), 415-65.

Williamson, Timothy (2013). Modal Logic as Metaphysics. Oxford University Press.

Wright, Crispin (1983). Frege's Conception of Numbers as Objects. Aberdeen University Press.

Wright, Crispin (2007). On Quantifying into Predicate Position. In Mary Leng, Alexander Paseau, and Michael Potter (Eds.), Mathematical Knowledge (150174). Oxford University Press. 